\title{
SERS spectroscopy for detection of hydrogen cyanide in breath from children colonised with P. aeruginosa
}

Lauridsen, Rikke Kragh; Skou, Peter Bæk; Rindzevicius, Tomas; Wu, Kaiyu; Molin, Søren; Engelsen, Søren Balling; Nielsen, Kim Gjerum; Johansen, Helle Krogh; Boisen, Anja

Published in:

Analytical Methods

Link to article, DOI:

10.1039/C7AY01693J

Publication date:

2017

Document Version

Peer reviewed version

Link back to DTU Orbit

Citation (APA):

Lauridsen, R. K., Skou, P. B., Rindzevicius, T., Wu, K., Molin, S., Engelsen, S. B., Nielsen, K. G., Johansen, H. K., \& Boisen, A. (2017). SERS spectroscopy for detection of hydrogen cyanide in breath from children colonised with P. aeruginosa. Analytical Methods, 9(39), 5757-5762. https://doi.org/10.1039/C7AY01693J

\section{General rights}

Copyright and moral rights for the publications made accessible in the public portal are retained by the authors and/or other copyright owners and it is a condition of accessing publications that users recognise and abide by the legal requirements associated with these rights.

- Users may download and print one copy of any publication from the public portal for the purpose of private study or research.

- You may not further distribute the material or use it for any profit-making activity or commercial gain

- You may freely distribute the URL identifying the publication in the public portal 


\title{
Surface-enhanced raman spectroscopy for detection of hydrogen cyanide in breath from children colonised with $P$. aeruginosa
}

\author{
Rikke Kragh Lauridsen ${ }^{a}$, Peter Bæk Skou ${ }^{b}$, Tomas Rindzevicius $^{\mathrm{a}}$, Kaiyu Wu ${ }^{\mathrm{a}}$, Søren Molin ${ }^{\mathrm{c}}$, Søren \\ Balling Engelsen ${ }^{\mathrm{b}}$, Kim Gjerum Nielsen ${ }^{\mathrm{d}}$, Helle Krogh Johansen ${ }^{\mathrm{e}^{\dagger}}$, Anja Boisen ${ }^{\mathrm{a}^{\dagger}}$ \\ There is a need for a fast and non-invasive tool to detect Pseudomonas aeruginosa airway colonisation in cystic fibrosis \\ (CF) patients unable to expectorate. Fifty CF children and 19 controls aged 5-17 years were included in the feasibility study. \\ A surface-enhanced Raman spectroscopy (SERS) nanochip optimised for detection of trace amounts of the $P$. aeruginosa \\ biomarker hydrogen cyanide (HCN) was mounted inside a Tedlar bag, which the patient breathed into. The SERS chip was \\ then analysed in a Raman spectrometer, investigating the $\mathrm{C} \equiv \mathrm{N}$ peak at $2131 \mathrm{~cm}^{-1}$ and correlated with sputum cultures. \\ One new $P$. aeruginosa colonisation occurred during the trial period. The $\mathrm{C} \equiv \mathrm{N}$ peak intensity was enhanced in this sample \\ in contrast to the subject's 3 other samples. Three additional patients had intense C=N SERS signals from their breath, but \\ no $P$. aeruginosa was cultured from their sputum. It is concluded that SERS spectroscopy can be developed into an easy to \\ use hypersensitive clinical prescreening method for detection of HCN in human breath.
}

\section{Introduction}

Pseudomonas aeruginosa airway infections are the major cause of morbidity and mortality in cystic fibrosis (CF) patients ${ }^{1,2}$. The methods that are being used in the CF clinic to discover $P$. aeruginosa in non-expectorating children are invasive, because either a swab needs to enter through the mouth and into the pharynx or a tube is inserted through the nose and into the larynx to obtain a sample. Even with these procedures there is a low sensitivity, and it has been estimated that approximately $75 \%$ of all newly discovered $P$. aeruginosa infections are already persistently established ${ }^{3}$. Therefore, there is an urgent need for a sensitive, fast, cheap and noninvasive diagnostic tool to detect early $P$. aeruginosa airway colonisation in CF children who are unable to produce sputum or expectorate properly. $P$. aeruginosa emits the poisonous gas hydrogen cyanide ( $\mathrm{HCN})$, which can be an advantage combating other microorganisms in the lungs ${ }^{4,5}$. HCN is considered a potential $P$. aeruginosa biomarker, and it was

\footnotetext{
a. DTU Nanotech, Technical University of Denmark, Ørsteds Plads 345B, 2800 Lyngby, Denmark.

b. Department of Food Science, University of Copenhagen, Rolighedsvej 26, 1958 Frederiksberg C, Denmark.

c. DTU Systems Biology, Technical University of Denmark, Matematiktorvet 301, 2800 Lyngby, Denmark.

d. Paediatric Pulmonary Service 5003, Copenhagen University Hospital,

Rigshospitalet, Blegdamsvej 9, 2100 København $\varnothing$, Denmark.

e. Department of Clinical Microbiology 9301, Rigshospitalet, Juliane Maries Vej 22, 2100 København $\varnothing$, Denmark

† Shared last authorship.

*Corresponding author email: hkj@biosustain.dtu.dk

Electronic Supplementary Information (ESI) available: [details of any supplementary information available should be included here]. See
} DOI: $10.1039 / \mathrm{x} 0 \times x \times 0000 \mathrm{x}$ detected at median values of $13.5 \mathrm{ppb}$ in children's breath using selected ion flow tube mass spectroscopy (SIFT-MS) ${ }^{4}$. In the SPACE study, Gilchrist and co-workers measured HCN in exhaled breath of CF children, also using SIFT-MS ${ }^{6}$. At present time, SIFT-MS has not been optimized to meet the need for fast detection of bacteria, and the measurements were made after up to 24 hours, whereby some of the exhaled HCN may have been lost. Additionally, in the SPACE study, chronically infected patients were included, whose $P$. aeruginosa may have ceased producing HCN. Previously we have shown that at some point during adaptation $P$. aeruginosa can stop producing $\mathrm{HCN}$, which can explain why the biomarker cannot be detected in the breath of all CF patients with a chronic airway infection ${ }^{7}$.

Between $\mathrm{C}$ and $\mathrm{N}$ of cyanide there is a triple bond, which gives rise to a distinct peak in a Raman spectrum at $2131 \mathrm{~cm}^{-1}$. Raman spectroscopy is a fast and non-invasive analytical technique extensively used for molecular fingerprinting. The technique identifies analytes by differentiating their molecular vibrational frequencies in the probed Raman scattering signal. However, the limit of detection for traditional Raman spectroscopy is in the range of millimolar concentration ${ }^{8}$, impeding the use of the technique for detecting molecules at lower concentrations. To indicate $P$. aeruginosa infection in the breath of patients with $\mathrm{CF}$, detection levels of $\mathrm{HCN}$ below $20 \mathrm{ppb}$ are required ${ }^{4}$. As an advance of Raman spectroscopy, surface-enhanced Raman spectroscopy (SERS) probes the enhanced Raman scattering signal from analytes sitting on the surface of noble metal nanostructures ${ }^{9}$. In addition, the affinity of cyanide towards gold $^{10}$ makes SERS very suitable for detecting trace amount of HCN. Recently, we have demonstrated the fast identification and quantification of gas- 
phase HCN down to $18 \mathrm{ppb}$ on gold coated silicon nanopillar substrates ${ }^{11}$, which are manufactured in large-format by a cost effective process ${ }^{12}$. We have also applied the SERS method to detect $\mathrm{HCN}$ in emissions from $P$. aeruginosa cultures isolated from CF patients ${ }^{7}$. Our results indicate that SERS on the nanopillar substrate may have significant potential towards fast, cheap and non-invasive detection of $P$. aeruginosa lung colonisation from the breath of children with CF. In this feasibility study, we wanted to use the gold $(\mathrm{Au})$-coated silicon (Si) SERS substrate for direct detection of HCN in the breath of CF children with an early $P$. aeruginosa colonisation.

\section{Materials and Methods}

\section{Subjects and study design}

Fifty children with CF (median age 11 years, range 5-17 years) and 19 non-CF, age-matched controls were included in the study. CF patients were excluded if their latest total IgG measured by an Enzyme-Linked-Immosorbent Assay (ELISA) against $P$. aeruginosa antigens was above 2.39 ELISA units, or if the number of precipitating antibodies against $P$. aeruginosa was $\geq 2$, meaning that they had a chronic $P$. aeruginosa infection $^{13}$, except from two CF patients who were included to compare in vitro results ${ }^{7}$ to in vivo breath analysis. A total of $50 \mathrm{CF}$ children were followed for four months at the CF centre at Rigshospitalet. Each month the child exhaled into a $3 \mathrm{~L}$ Tedlar bag with the SERS substrate mounted inside, which was analysed a few hours later. The intensity of the cyanide peak at $2131 \mathrm{~cm}^{-1}$ was compared to culture of sputum samples obtained via expectoration or endo-laryngeal suction.

The controls were patients and siblings seen in the CF outpatient clinic at Rigshospitalet during the study period, which was January-April 2016. Controls were not included if they had CF, primary cilia dyskinesia (PCD) or asthma. Enrolment took place during the entire study period, and each subject was tested between 1 and 5 times. Controls were only tested once. The controls only delivered breath and no sputum sample.

\section{The SERS substrate}

The disposable SERS substrate was fabricated on a single-side polished Si wafer using $3 \mathrm{~min}$ reactive ion etching (RIE) with alternating $\mathrm{SF}_{6}$ bombardment and $\mathrm{O}_{2}$ passivation for protection of the emerging nanopillars, followed by a $1 \mathrm{~min} \mathrm{O}_{2}$ plasma cleaning step. Up till 5 days prior to use, Au was evaporated onto the Si nanopillars, forming $225 \mathrm{~nm}$ caps used for SERS detection (Figure 1a). On the day of exposure the wafer was cut into $5 \times 10 \mathrm{~mm}^{2}$ substrates using a diamond cutter and tweezers. The substrates were cleaned by immersion into ethanol (Absolute grade, CHROMASOLV $\mathrm{R}$, Sigma-Aldrich, St. Louis, MO, USA) for 3 min followed by $\mathrm{H}_{2} \mathrm{O}$ (Molecular Biology Reagent grade, Sigma-Aldrich, St. Louis, MO, USA) for $3 \mathrm{~min}$ and left to dry on a tissue whereby the pillars would lean to enable SERS detection (Figure 1b). For easy handling and transportation the SERS substrate was mounted inside a small Petri dish, using double sided adhesive tape (Scotch), with a Post-It on top, the tacky side facing up for holding the substrate. The wall of the Petri dish protected the delicate SERS substrate so it would not get in contact with the Tedlar bag and be scratched (Figure 1c).

\section{The collection device}

Three $\mathrm{L}$ disposable Tedlar sample bags with polypropylene fittings (SKC, Pennsylvania, USA) were used for breath collection, with one large corner cut off, so the Petri dish containing the SERS substrate could be mounted inside (Figure 1d). The bag was closed with a clamp (Scentroid, Ontario, Canada). Part of a disposable latex tube (Philips Respironics, Colorado, USA) was used to connect the inlet of the Tedlar bag to the PEP flutter (Intersurgical, Berkshire, UK) that the child would blow through, equipped with a $3.5 \mathrm{~mm}$ resistance (Wellspect Healthcare, Mölndal, Sweden).

\section{Sample collection}

The child filled the Tedlar bag with 1-5 deep exhalations. The bag was closed and left for 15 minutes before evacuation in a laminar air flow bench. The lid was put on the Petri dish, which was then secured tightly in a small zipper bag for transportation. A short questionnaire was also filled in, regarding present well-being, coffee intake and smoking, because it is known that these stimulants can influence the amount of $\mathrm{HCN}$ in the breath ${ }^{14,15}$.

\section{Raman measurements}

SERS measurements were recorded using an FT-Raman instrument (Bruker VERTEX 70, Bruker Optik, Ettlingen, Germany), equipped with a $1064 \mathrm{~nm}$ laser and an indium gallium arsenide (InGaAs) detector. Samples were measured in a 180 degrees backscattering geometry using a laser power of $200 \mathrm{~mW}$ with 32 scans at a resolution of $4 \mathrm{~cm}^{-1}$. Each sample was measured at 5 different positions on the substrate.

\section{Data analysis}

The five spectra obtained from different positions on the SERS substrate were averaged in order to increase the signal to noise ratio. The average per sample was subsequently preprocessed using Standard Normal Variate $(\mathrm{SNV})^{16}$. SNV eliminates off-sets and slopes from spectra with similar spectral features aiding visual inspection as well as reducing complexity of later data analysis. Principal component analysis $(\mathrm{PCA})^{17}$ was performed using the PLS_toolbox (Ver. 7.9.5, Eigenvector Research, USA) in Matlab 2015a (MathWork, USA) on the pre-processed data both on the full spectral range and a reduced data set with only the $\mathrm{HCN}$ peak around $2131 \mathrm{~cm}^{-1}$. In figures 2 and 3 , the background was subtracted and spectra were shifted up for clarity.

\section{Ethical approval}

The local ethics' committee approved the feasibility study, which was assigned the number H-15016027. Informed consent was obtained from the parents or from the children aged 15 years or older. 


\section{Results}

\section{Demographics}

A total of $50 \mathrm{CF}$ patients and 19 controls were included in the study (Table 1). With 4 months and $50 \mathrm{CFs}$, about 2 new cases of $P$. aeruginosa colonisation were expected (according to the outcome of ${ }^{6}$ ), but a few patients with chronic infections were included, leaving fewer naïve cases.

Table 1. Demographics of included CF patients and controls

CF patients
\begin{tabular}{lcc}
\hline Median age, years (range) & $11(5-17)$ & \\
\hline Gender, number (\%) & Males 23 (46) & Females 27 (54) \\
\hline No. of visits by CF patients in total: 87 & \\
\hline
\end{tabular}

Controls

Median age, years (range) $8(5-17)$

Gender, number (\%) Males 12 (63) $\quad$ Females 7 (37)

No. of visits by the controls in total: 19

Underlying diseases of the controls: Other phacomatoses, Secondary pulmonary condition, Cough, Pneumonia, Pulmonary

atelectasis, Stridor, Pulmonary hemoptysis, Acute bronchitis.

\section{Comparison of in vitro and in vivo SERS}

Previously, in vitro SERS measurements were made on $P$. aeruginosa cultures isolated from chronically infected paediatric CF patients in the outpatient clinic ${ }^{7}$. Two of these patients were also included in the study because their isolates represented two different trends in the in vitro study. In the in vitro study, all first (wild type-like) isolates emitted detectable $\mathrm{HCN}$. After it had turned into a chronic infection, subject $A$ had a las $R$ mutated $P$. aeruginosa isolate which still produced $\mathrm{HCN}$ (Figure 2a, red spectrum), while subject $\mathrm{B}^{\prime} \mathrm{s}$ las $R$ mutated $P$. aeruginosa isolate had turned off the expression of $\mathrm{HCN}$ (Figure 2b, red spectrum). Therefore, it was interesting to investigate whether this trend was also seen in vivo. The isolates were obtained five years before, so the bacteria could possibly have mutated further in the meantime. As seen in the figures' blue spectra, no HCN was detected from their breath. Patient A: Both the wild type-like (green) and the las $R$ mutated isolate (red) emitted $\mathrm{HCN}$, which can be seen from the intense $\mathrm{C} \equiv \mathrm{N}$ peak at $2131 \mathrm{~cm}^{-1}$, whereas no $\mathrm{HCN}$ was detected from the patients' breath (blue). Patient $\mathrm{B}$ : The wild type-like isolate emitted $\mathrm{HCN}$, which did neither the lasR mutated isolate, nor the breath sample. SERS on headspace of Luria Broth (LB) growth medium is included as reference (black). There is a background peak in the SERS spectra close to where the cyanide peak is located.

Although chronically infected with $P$. aeruginosa, patient A only cultured Stenotrophomonas maltophilia and no $P$. aeruginosa from sputum, whereas patient B cultured both mucoid and non-mucoid $P$. aeruginosa.

Four cases of elevated cyanide signal
There was one new case of $P$. aeruginosa during the 4 months' trial period. Patient $C$ was a 15 year-old male with a positive sputum culture at his $3^{\text {rd }}$ out of 4 visits during the four months. Figure 3 shows the SERS spectra from his four visits, with a more intense triple bond signal about $2131 \mathrm{~cm}^{-1}$ from visit no. 3. Additionally, the red peak is slightly shifted towards higher wave numbers, away from the background signal. After antibiotic eradication therapy between the third and fourth visit to the outpatient clinic, the background peak is back to where it started (green). The intensity is increased at visit no. 3 , and the peak is slightly shifted towards higher wave numbers.

Three additional times during the trial period the SERS triple bond signal was elevated in breath samples,but none of these patients had $P$. aeruginosa cultured from their sputum. In one of the cases, the Tedlar bag had been left for $60 \mathrm{~min}$ before the SERS substrate was taken out, and it turned out that this had also been the case in the $3^{\text {rd }}$ visit of patient $C$, and it is uncertain whether this had an effect on the peak intensity. Two of the incidents were from children whose parents were smoking in-door at home, which may have been detected on the chip. One of them cultured yeast, while the other only cultured non-pathogenic oropharyngeal microorganisms from the sputum samples.

\section{Discussion}

We explored whether the SERS chip could be used to detect the biomarker $\mathrm{HCN}$ in the breath of CF children with an early $P$. aeruginosa airway colonisation and whether our findings could be correlated to the findings in sputum cultures. Due to a limited number of includable patients, only one new case of $P$. aeruginosa was cultured during the study period, and it was most likely detected on the SERS chip. In the SERS measurements from the patient's other visits, only the background peak was present. Three additional cases of intense $\mathrm{CN}$ signal occurred in children, whose sputum cultures were $P$. aeruginosa negative. There was a dependency of exposure time on peak intensity, and it seemed like passive smoking could also be detected from the children's breath.

The reason to include two patients with chronic infections was that we wanted to compare our in vitro results ${ }^{7}$ on their first colonising strain and later lasR mutated isolate to in vivo breath analysis, because these cannot be expected to be the same ${ }^{18}$. Patient A, whose las $R$ mutated $P$. aeruginosa isolate emitted $\mathrm{HCN}$, was 8 years old; and at the time the strain was isolated she was 3. An explanation to why no $\mathrm{HCN}$ was detected in the breath of this patient could be that the bacteria had mutated even further since the isolate was cultured back in 2011. Even though this CF patient has about 25 precipitating antibodies against $P$. aeruginosa today, no $P$. aeruginosa had been cultured from her last 3 sputum samples. Only when naso-laryngeal suction was performed, mucoid $P$. aeruginosa was cultured; and it can be questioned whether it came from the patient's sinuses, and also whether young patients are able to expectorate properly. 
In the $P$. aeruginosa case cultured during the trial, the position of the Raman peak was not outside the normal shift seen across patients; however, the patient specific shift (assuming a given patient has an individual peak position) can be an indication. More similar cases are necessary to investigate or reject this hypothesis. Two of the 3 additional patients with positive cyanide SERS signal were children whose parents were smoking in-door at home. The third patient had an immediate microscopy result indicating few Gram-negative rods, but when the final microbiological result came, it was Moraxella catarrhalis, which is a diplococcus, that can hardly be mistaken for a Gram-negative rod. The question is, whether Gramnegative rods were present in the beginning, but not cultured subsequently. Another observation was, that these children can be exposed to passive smoking as they enter the CF centre, which is located on the main floor, where people are standing right outside, smoking, because this is prohibited inside the hospital.

A major drawback of the applied method is the background signal on the Au SERS substrate, which has also been reported elsewhere ${ }^{21}$, and it seems to be enhanced by the warm and moist breath. The three cases with intense triple bond signal and negative sputum culture were followed for five months after study termination, and none of them cultured $P$. aeruginosa during the follow-up period, suggesting the SERS results must have been false positives. Regarding the control subjects, these need to be selected more carefully. Some were too sick, and some could have had $P$. aeruginosa in their lungs, since their sputum was not cultured. Ideally, the study period should have been longer or more patients should have been included to obtain more new positive $P$. aeruginosa cultures. In the SPACE study, Gilchrist and co-workers followed $233 \mathrm{CF}$ children for 24 months, and 71 children cultured $P$. aeruginosa positive. With $50 \mathrm{CF}$ children followed for 4 months, an estimate of two new $P$. aeruginosa cases were expected. To ease logistics and keep it to one centre, primary ciliary dyskinesia (PCD) patients could have been included, too, because it is known that the evolution of $P$. aeruginosa follows the same mutational steps in $\mathrm{PCD}$ as in $\mathrm{CF}^{22}$.

In the SPACE study, 21 chronically infected patients were included, who had not been cultured positive in their swab or sputum samples for the last 12 months $^{6}$, and it can be questioned what the probability would be to culture positive during the trial period. In future studies we will suggest to consider using a more sensitive reference method, such as PCR instead of just sputum culture to detect whether $P$. aeruginosa is present in the sputum sample or not. Another issue regarding bacterial culturing is the transportation conditions of the sputum samples which could be optimised to better secure survival of the microorganisms. Samples should be either cooled down or kept at $37^{\circ} \mathrm{C}$ and transported immediately to the clinical microbiology laboratory and not wait up till 24 or 48 hours due to internal mail delivery. The PCA analyses did not show strong trends in the pre-processed spectral data, and it is important to remember that the spectral measurements as well as the sputum culturing results carry large uncertainties due to exposure time differences, delayed analysis of sputum samples, possibly leading to increased number of false negative results, etc., making it challenging to relate the two.

The $\mathrm{CN}$ peak intensity seemed to depend on exposure time, and in future studies the device could be left for e.g. 60 minutes for complete exposure to occur. In the in vitro study, 15 min exposure time was applied, which was sufficient, and it is a learning to take to the next clinical trial. Another way to overcome the exposure time issue could be to condensate the breath directly onto the SERS substrate. Disposable devices for this are available (like the R-Tube from MESM Ltd.) ${ }^{19}$, but it is uncertain if patient compliance would be affected by having to breathe into a "chimney" device like this instead of into the applied "balloon-like" device which most of the children thought was fun. It could also be considered to make the substrate alkaline, so that a maximal amount of the cyanide would be expected to stay on the Au surface (according to Henry's Law ${ }^{20}$ ).

\section{Conclusions}

The present study, has demonstrated that Surface Enhanced Raman Spectroscopy (SERS) can be developed into a very useful and sensitive method for early detection of $P$. aeruginosa in CF patients. However, the method is highly sensitive to other sources of $\mathrm{HCN}$ wherefore more $P$. aeruginosa positive patients must be tested in future trials in order to prove whether this method can be used for diagnosis.

\section{Acknowledgements}

We would like to thank all CF patients and controls and parents who took part in the study. We would also like to acknowledge Michael Stenbæk Schmidt for inventing the first nanopillar SERS substrate, and to thank Flemming Larsen for seeing the opportunity of forming this CF project.

\section{Funding}

This work was supported by The Danish Council for Independent Research's Sapere Aude project "NAPLAS", by the Danish National Research Foundation (DNRF122) and Villum Fonden (Grant No. 9301). HKJ was funded by a clinical research stipend from The Novo Nordisk Foundation and Rigshospitalets Rammebevilling 2015-17 and Lundbeck Foundation Grant R167-2013-15229. KGN was founded by The Research Committee, Rigshospitalet.

\section{References}

1 R. L. Gibson, J. L. Burns, B. W. Ramsey, Am J Respir Crit Care Med, 2003, 168, 918-951.

2 C. Koch, N. Høiby, Respiration, 2000, 67, 239-247.

3 H. Johansen, L. M. Sommer, R. Marvig, L. Jensen, M. Skov, T. Pressler, S. Molin, Pediatr Pulmonol, 2015, 50(41), 77-107. 
4 B. Enderby, D. Smith, W. Carroll, W. Lenney, Pediatr Pulm, 2009, 44, 142-147.

5 D. Smith, P. Spanel, F. J. Gilchrist, W. Lenney, J Breath Res., 2013, 7, 1-13.

6 F. Gilchrist, J. Belcher, A. Jones, D. Smith, A. Smyth, K. Southern, P. Spanel, A. Webb, W. Lenney, Eur Resp J, 2015, 1 (00044), 1-8.

7 R. K. Lauridsen, L. M. Sommer, H. K. Johansen, T. Rindzevicius, S. Molin, L. Jelsbak, S. B. Engelsen, A. Boisen, Sci Rep, 2017

8 K. Cho, Y. S. Jang, M. Gong, K. Kim, S. Joo, Appl Spectrosc, 2002, 56, 1147-1151.

9 K. A. Willets, R. P. van Duyne, Annu Rev Phys Chem, 2007, 58, 267-297.

10 C.-Y. Liu, W.-L. Tseng, Chem Commun, 2011, 47, 2550-2552.

11 R. K. Lauridsen, T. Rindzevicius, S. Molin, H. K. Johansen, R. W. Berg, T. S. Alstrøm, K. Almdal, F. Larsen, M. S. Schmidt, A. Boisen, Sens Biosens Res, 2015, 5, 84-89.

12 M. S. Schmidt, J. Hübner, A. Boisen, Adv Mat, 2012, 24, OP11-OP18.

13 T. Pressler, F. Karpati, M. Granström, P. K. Knudsen, A. Lindblad, L. Hjelte, H. V. Olesen, P. Meyer, N. Høiby, J Cyst Fibros, 2009, 8(1), 37-42.

14 R. K. Lauridsen, PhD thesis, Technical University Denmark, 2016.

15 F. M. Schmidt, M. Metsälä, O. Vaittinen, L. Halonen, J Breath Res, 2011, 5, 046004.

16 R. J. Barnes, M. S. Dhanoa, S. J. Lister, Appl Spectrosc, 1989, 43, 772-777

17 S. Wold, K. Esbensen, P. Geladi, Chemom. Intell. Lab. Syst., 1987, 2, 37-52

18 R. Bro, A. K. Smilde, Anal Methods, 2014, 6, 2812-2831.

19 J. Zhu, H. D. Bean, M. J. Wargo, L. W. Leclair, J. E. Hill, J Breath Res, 2013, 7, 016003.

20 R-Tube from MESM Ltd. https://www.mesm.com/products/spirometers-andrespiratory-monitors/r-tube-starter-kit-100 (accessed 30/6 2017).

21 J. Ma, P. K. Dasgupta, Environm Sci Techn, 2010, 44, 30283034.

22 W. Premasiri, R. Clarke, S. Londhe, M. Womble, J Raman Spectrosc, 2001, 32, 919-922.

23 L. M. Sommer, M. C. Alanin, R. L. Marvig, K. G. Nielsen, B. Høiby, C. von Buchwald, S. Molin, H. K. Johansen, Sci Rep, 2016, 6, 28732. 
Au nanopillars

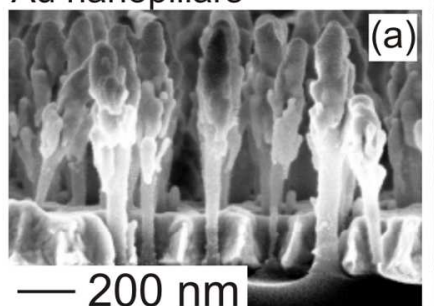

Leaning Au nanopillars
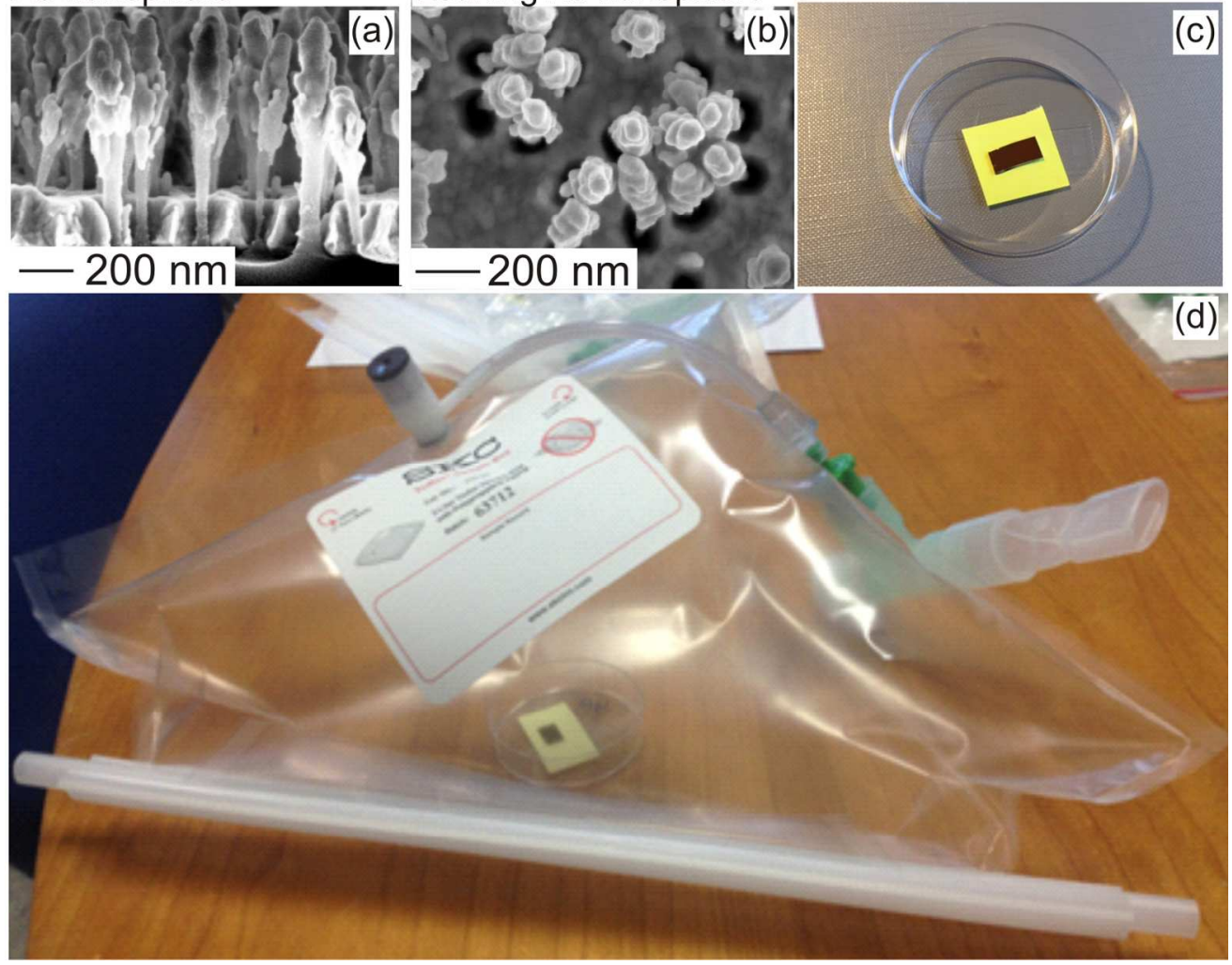

The SERS substrate. a) Scanning Electron Microscopy (SEM) image of the vertically standing nanopillars prior to leaning. The Si nanopillars are capped by gold, and the underlying Si substrate is covered by a gold film. b) Top-view SEM image of two clusters of nanopillars after leaning. c) Petri dish with a gold (Au) SERS substrate mounted inside. d) The filled collection device with the SERS chip inside, being exposed to exhaled breath. The tip of the PEP flutter is seen to the right. The fitting on top and the clamp at the bottom keep the Tedlar bag closed. 

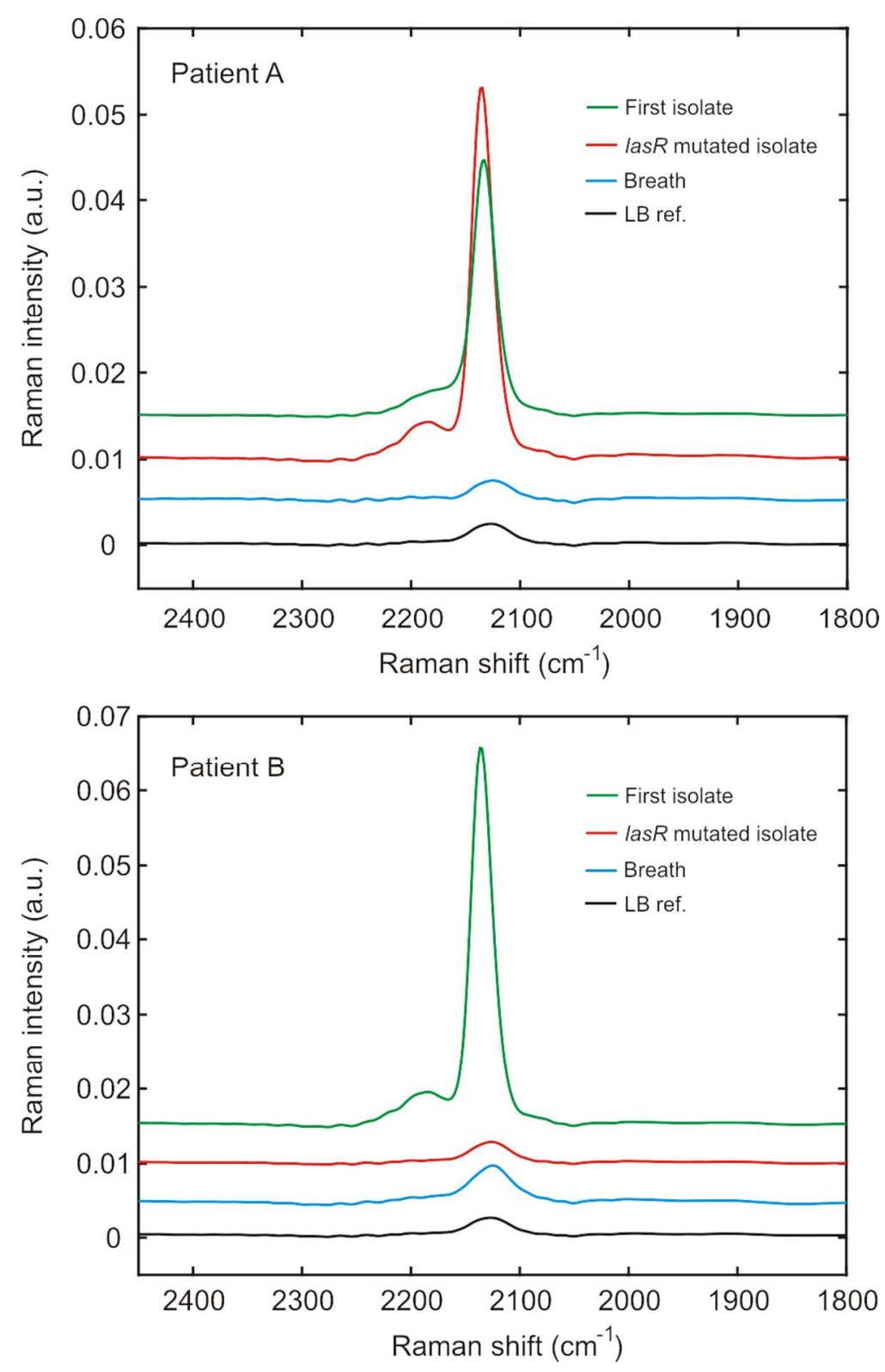

In vitro and in vivo SERS on P. aeruginosa isolates and breath from two CF patients who today are chronically infected. Patient A: Both the wild type-like (green) and the lasR mutated isolate (red) emitted $\mathrm{HCN}$, which can be seen from the intense $\mathrm{C} \equiv \mathrm{N}$ peak at $2131 \mathrm{~cm}-1$, whereas no $\mathrm{HCN}$ was detected from the patients' breath (blue). Patient B: The wild type-like isolate emitted HCN, which did neither the lasR mutated isolate, nor the breath sample. SERS on headspace of Luria Broth (LB) growth medium is included as reference (black). There is a background peak in the SERS spectra close to where the cyanide peak is located. 


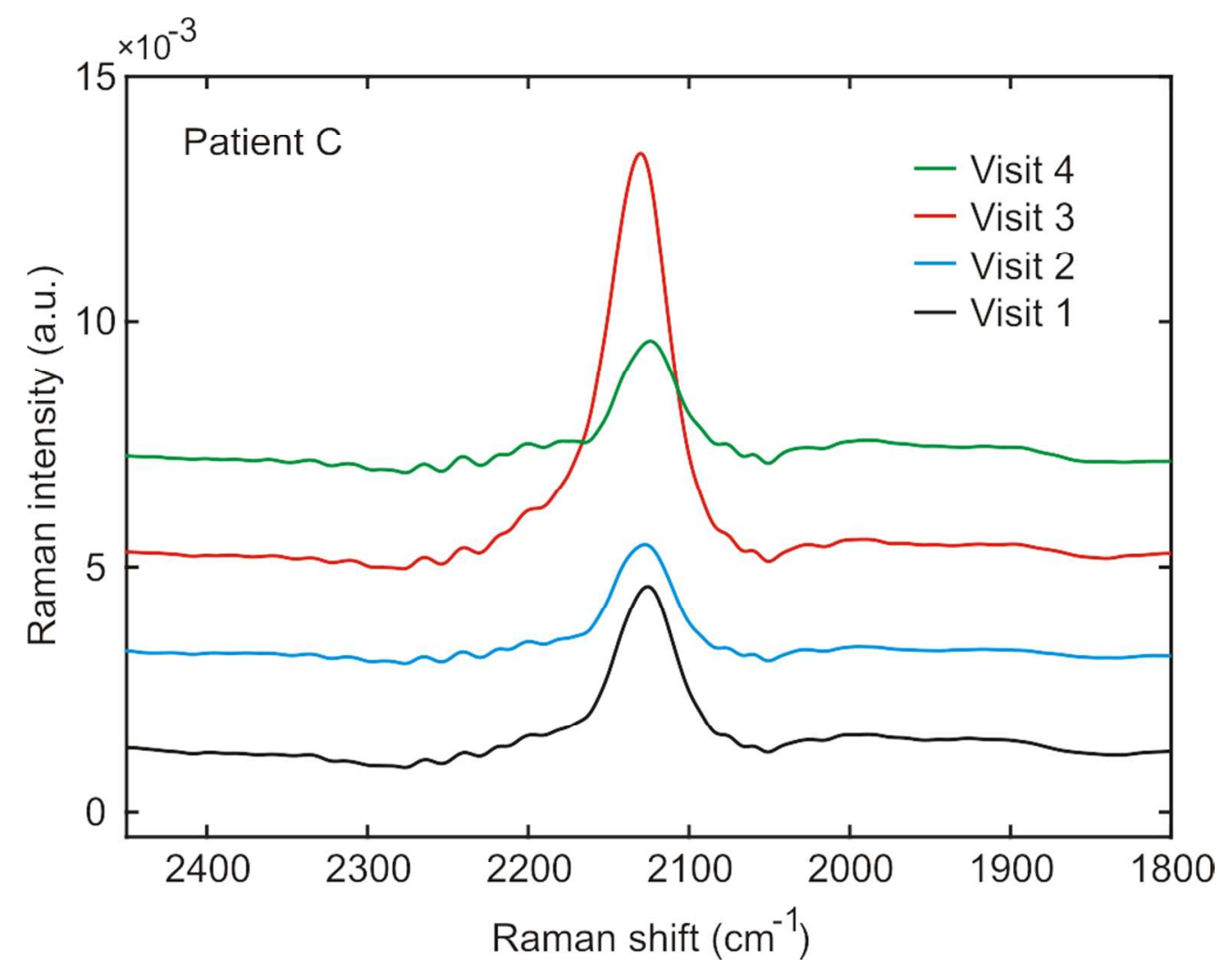

Zoom on the triple bond peak about $2131 \mathrm{~cm}-1$ from patient C's visits. The intensity is increased at visit no. 3 , and the peak is slightly shifted towards higher wave numbers. 